\title{
PERCEPÇÃO E RELATO DE DISCENTES FRENTE ÀS AVALIAÇÕES VIRTUAIS EM MULHERES NO CLIMATÉRIO
}

Percepción y reporte de estudiantes ante las evaluaciones virtuales en mujeres en el climaterio Perception and report of students facing virtual evaluations of women in the climacteric

\section{Maria Amélia Pires Soares da Silva ${ }^{1}$, Viviane Jerônimo de Macêdo $^{2}$, Ana Caroline da Fonseca Nunes ${ }^{3}$, Ana Caroline de Araújo Silva ${ }^{4}$, Laiane Santos Eufrásio ${ }^{5}$}

\section{RESUMO}

Devido ao isolamento causado pela pandemia do novo coronavírus, as instituições de ensino superior tiveram de moldar suas atividades, adaptando-as para o formato remoto. O presente trabalho tem como objetivo relatar a percepção de discentes da graduação em Fisioterapia e Nutrição sobre as avaliações virtuais em mulheres na fase do climatério. Trata-se de um estudo descritivo, do tipo relato de experiência, com recorte pertencente a um projeto de extensão e pesquisa. Participaram das ações uma docente efetiva da área de Fisioterapia em Saúde da mulher, dois discentes do curso de Fisioterapia, dois discentes do curso de Nutrição e 40 mulheres climatéricas. Avaliações virtuais foram realizadas por meio das plataformas de comunicação Google Meet e WhatsApp. A estratégia de continuidade do projeto foi extremamente importante para a experiência acadêmica por simplificar a linguagem científica e ampliar os conceitos de educação em saúde, devido à troca de informações estabelecida entre o avaliador e a participante.

Palavras-chave: Climatério; Avaliação de sintomas; Avaliação em Saúde.

\footnotetext{
${ }^{1}$ Graduanda em Fisioterapia pela Universidade Federal do Rio Grande do Norte (UFRN)/Faculdade de Ciências da Saúde do Trairi (FACISA).

${ }^{2}$ Graduanda em Fisioterapia pela Universidade Federal do Rio Grande do Norte (UFRN)/Faculdade de Ciências da Saúde do Trairi (FACISA)

${ }^{3}$ Graduanda em Nutrição pela Universidade Federal do Rio Grande do Norte (UFRN)/Faculdade de Ciências da Saúde do Trairi (FACISA).

${ }^{4}$ Graduanda em Nutrição pela Universidade Federal do Rio Grande do Norte (UFRN)/Faculdade de Ciências da Saúde do Trairi (FACISA).

${ }^{5}$ Professora Doutora de Fisioterapia na Saúde da Mulher e Dermatofuncional da Faculdade de Ciências da Saúde do Trairi - FACISA, Universidade Federal do Rio Grande do Norte (UFRN). Santa Cruz/RN.
} 


\section{RESUMEN}

Debido al aislamiento provocado por la nueva pandemia de coronavirus, las instituciones de educación superior tuvieron que dar forma a sus actividades, adaptándolas al formato remoto. Este estudio tiene como objetivo reportar la percepción de estudiantes de pregrado en Fisioterapia y Nutrición sobre las evaluaciones virtuales en mujeres en la fase climatérica. Se trata de un estudio descriptivo, tipo de informe de experiencia, con recorte perteneciente a un proyecto de extensión e investigación. En las acciones participaron una docente eficaz en el área de Fisioterapia en Salud de la Mujer, dos estudiantes del curso de Fisioterapia, dos estudiantes del curso de Nutrición y 40 mujeres climatéricas. Se realizaron evaluaciones virtuales a través de las plataformas de comunicación Google Meet y WhatsApp. La estrategia de continuidad del proyecto fue de suma importancia para la experiencia académica ya que simplificó el lenguaje científico y amplió los conceptos de educación en salud, debido al intercambio de información establecido entre el evaluador y el participante.

Palabras clave: Climaterio, Evaluación de Síntomas; Evaluación en Salud.

\section{ABSTRACT}

Due to the isolation caused by the new coronavirus pandemic, higher education institutions had to shape their activities, adapting them to the remote format. This study aims to report the perception of undergraduate students in Physical Therapy and Nutrition about virtual assessments in women in the climacteric phase. This is a descriptive study, type of experience report, with clipping belonging to an extension and research project. Participated in the actions an effective teacher in the area of Physiotherapy in Women's Health, two students from the Physiotherapy course, two students from the Nutrition course and 40 climacteric women. Virtual assessments were carried out through the communication platforms Google Meet and WhatsApp. The project's continuity strategy was extremely important for the academic experience as it simplified the scientific language and expanded the concepts of health education, due to the exchange of information established between the evaluator and the participant.

Keywords: Climacteric; Symptom assessment; Health Evaluation. 


\section{INTRODUÇÃO}

No início de 2020, a população passou por diversas transformações mundiais, decorrentes da pandemia do novo coronavírus (COVID-19). O distanciamento social exigido pela situação pandêmica refletiu no cenário universitário e na assistência à saúde oferecida pelas instituições de ensino superior (EIS). As atividades de extensão universitária e pesquisa tiveram de ser adaptadas para acontecer de maneira remota, através do auxílio das tecnologias de informação e comunicação (TICs), de modo que fossem respeitados os protocolos de segurança e prevenção contra a contaminação pela COVID-19 (GUSSO et al., 2020; DINIZ et al., 2020; MARTINES, 2018).

O contexto afetou todas as faixas etárias, inclusive as mulheres que estavam passando pelo climatério. O fechamento dos espaços recreativos causou diversas alterações físicas e emocionais para esse público, entre elas podemos listar: a intensificação das manifestações clínicas e fisiológicas dos sintomas, a diminuição da prática de atividade física, a elevação dos níveis de estresse e ansiedade, assim como a recusa de uma alimentação saudável (KAYGISIZ et al., 2020; NIENHUIS CP, LESSER IA, 2020).

A teleconsulta e o telemonitoramento surgem como um método novo e importante para o modelo social de saúde (MATA et al., 2020). O ambiente virtual foi considerado um agente facilitador no que concerne o favorecimento da utilização das TICs nas avaliações fisioterapêuticas e nutricionais digitais, bem como a disponibilização de orientações e intervenções plausíveis de serem feitas remotamente, no contexto de cada participante, no seu ambiente domiciliar.

Logo, vê-se que essa modalidade por meio de tecnologia pode ser aplicado à população de mulheres climatéricas, visando a diminuição dos sintomas e dos efeitos negativos da inatividade, na prevenção das doenças crônicas não transmissíveis, como também na promoção à saúde emocional (MONTILLA, ALDRIGHI e MARUCCI, 2004; BRASIL, 2008; CONTE; FRANZ, 2015; FERREIRA et al., 2020). Além disso, tem sido um método aplicado nas universidades que vem fortalecendo vínculos entre comunidade e instituição e enriquecendo a vivência dos estudantes com a prática (MAGALHÃES et al., 2020). Desse modo, ressaltamos a necessidade da utilização de estratégias virtuais que simplificam as ações de promoção à saúde voltadas para as demandas referidas por essas mulheres nessa fase do ciclo da vida.

\section{OBJETIVO}

O trabalho teve como objetivo relatar a percepção de discentes da graduação em Fisioterapia e Nutrição sobre avaliações virtuais de mulheres na fase do climatério. 


\section{METODOLOGIA}

Trata-se de um estudo descritivo, do tipo relato de experiência, referente às avaliações virtuais de mulheres no climatério. O recorte pertence a um projeto de extensão e pesquisa, intitulado "Florescer: Fisioterapia e Práticas Corporais para mulheres no climatério" idealizado por uma docente efetiva da área de Fisioterapia em Saúde da mulher junto com estudantes do curso de Fisioterapia e Nutrição, de variados períodos dos cursos, todos da Universidade Federal do Rio Grande do Norte - Faculdade de Ciências da Saúde do Trairi (UFRN/FACISA), Campus Santa Cruz/RN.

Para este presente relato, participaram os envolvidos no processo de avaliação dos projetos, sendo dois discentes do curso de Fisioterapia (D1, D2) e dois discentes do curso de Nutrição (D3, D4). Os projetos surgiram durante a pandemia da COVID-19 após a necessidade de estudos sobre estratégias terapêuticas, de modo virtual, para amenizar a sintomatologia climatérica. Foi feito uma análise nas bases de dados e, tanto o Pilates em Solo, quanto a Hatha Yoga, foram as intervenções escolhidas por serem atividades de fácil aprendizagem e comuns na sociedade.

O estudo foi aprovado pelo Comitê de Ética e Pesquisa (CAAE: 33351920.0.0000.5568) em Junho de 2020 mas, devido a pandemia, as atividades acadêmicas presenciais precisaram reinventar-se, assim como a estrutura e o formato das intervenções das pesquisas. Dessa forma, pensando no bem-estar dos envolvidos e na prevenção do contágio, o projeto foi reformulado para o formato híbrido, sendo a maior parte remota (aplicação da ficha de avaliação e questionários; intervenções de Yoga, Pilates e Educação em Saúde) e, uma outra parte, necessariamente presencial (avaliação do assoalho pélvico).

Nos primeiros meses de 2021 iniciou-se a divulgação para recrutamento das participantes. O plano de divulgação foi realizado tanto de forma virtual (folder virtual, podcast para rádio), como também de forma presencial, nas Unidades de Saúde do município de Santa Cruz/RN. Foram convidadas mulheres com idade entre 40 e 65 anos, residentes do interior do Estado do Rio Grande do Norte (RN), que não tinham contraindicações para realizar atividades físicas, nem realizado tratamento de fisioterapia pélvica, que tinham afinidade com as práticas corporais e que assinaram o Termo de Consentimento Livre Esclarecido (TCLE).

Foi realizada uma capacitação para os pesquisadores/discentes envolvidos, objetivando a apresentação dos questionários e das escalas, além de padronização da fala para evitar vieses nas avaliações. O treinamento foi totalmente virtual, totalizando dois encontros, em que detalhadamente estudaram cada tópico dos questionários e escalas, assim como tiraram dúvidas em tempo real, caso necessário. Por meio de duas plataformas de comunicação, Google Meet e WhatsApp, realizaram a capacitação com dados teóricos e demonstrações práticas. Ao final, os participantes/pesquisadores 
conseguiram adquirir conhecimento, destreza e profissionalismo para iniciar e dar prosseguimento às avaliações. O fluxo que seguiu o projeto está representado na Figura 1 abaixo.

Figura 1 - Fluxograma do projeto.

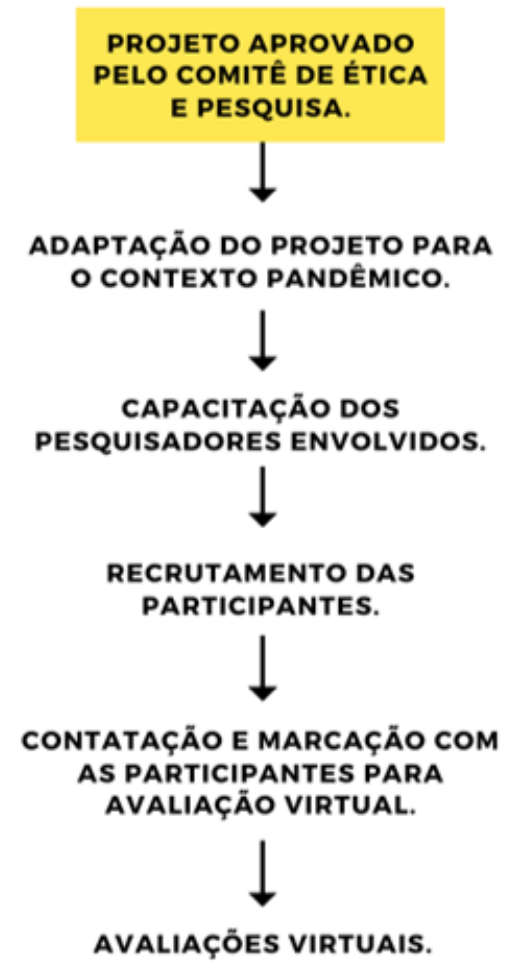

Fonte: Elaborado pelos autores.

Todos os discentes ficaram responsáveis por contactar as participantes e marcar as avaliações por meio de plataformas virtuais de áudio e vídeo, que permitiriam uma melhor compreensão por parte da voluntária para responder às questões. Foram utilizados os seguintes instrumentos de avaliação: Questionário de imagem corporal - Body Attitudes Questionnaire; WHO Disability Assessment Schedule (WHODAS 2.0); Índice de Função Sexual Feminina (FSFI); questionário Utian Quality of Life Scale (UQOL); Escala Climatérica de Greene; Menopause Rating Scale; Stages of Reproductive Aging Workshop (STRAW) e questionário de consumo alimentar.

O presente trabalho é um desenho da primeira etapa do projeto, com enfoque nas avaliações virtuais que ocorreram durante o mês de Junho e Julho de 2021. Para realização destas, também foram utilizadas as plataformas Google Meet e WhatsApp, por serem bem conhecidas no meio social e educacional, além de serem gratuitas, facilitando o manuseio e utilização. Pensando no conforto da entrevista e na manutenção do sigilo das informações, inicialmente foi sugerido para a voluntá- 
ria o uso de fones de ouvidos, assim como também foi questionado se o ambiente estava adequado para responder algumas perguntas. Também foi dito o tempo máximo de avaliação e a quantidade de questionários, prevenindo exaustões físicas e mentais.

Em cada avaliação estavam presentes um discente de Fisioterapia, um discente de Nutrição, a professora orientadora e a participante. O registro das respostas foi feito pelo aplicativo Google Forms e em seguida os dados foram reunidos em uma planilha do Google Sheets, com acesso restrito aos avaliadores. As avaliações virtuais seguiam as seguintes etapas:

1. Apresentação dos pesquisadores, seguido de apresentação do projeto;

2. Envio do Termo de Consentimento Livre e Esclarecido para leitura e assinatura da participante;

3. Breve educação em saúde sobre o climatério, seus sintomas e os possíveis tratamentos;

4. Captação de dados da voluntária, aplicação de questionários;

5. Orientação das próximas etapas do projeto;

6. Disponibilização de tempo para possíveis dúvidas, esclarecimentos.

Logo, além da coleta de dados das participantes, os integrantes também se colocaram na posição de educandos, compreendendo os diferentes impactos da pandemia na sintomatologia climatérica dessas mulheres e orientando o que estava ao alcance para auxiliar e direcionar melhor essas voluntárias.

\section{RESULTADOS E DISCUSSÃO}

Considerando as avaliações realizadas, os quatro discentes envolvidos no projeto obtiveram a experiência de avaliar virtualmente 40 mulheres climatéricas, percebendo que o perfil de idade das participantes variou entre 41 e 60 anos, a maioria eram procedentes do município de Santa Cruz/RN e com queixas urinárias presentes, assim como outras relacionadas ao próprio período do climatério.

Segundo visão geral dos avaliadores de ambos os cursos, a experiência foi bem diversificada. As escalas e questionários da Fisioterapia descreveram o perfil ginecológico obstétrico e analisaram a prevalência da sintomatologia climatérica e das disfunções do assoalho pélvico. Já o questionário 
da Nutrição avaliou os hábitos e estilo de vida das participantes. Sendo assim, as percepções das discentes frente aos diferentes achados entre as avaliações das duas áreas da saúde e os aprendizados quanto a esta modalidade de avaliação é de extrema importância para explanar.

\section{SOB AS PERSPECTIVAS DAS DISCENTES DE FISIOTERAPIA}

Estiveram presentes duas discentes do curso de Fisioterapia revezando entre as avaliações fisioterapêuticas virtuais, juntamente com as de Nutrição. Para a coleta das experiências nas avaliações de cada uma, foram colhidos trechos dos relatos por meio de um grupo de WhatsApp, após as experiências em questão.

A seguir, alguns relatos dos discentes:

"Avaliar vitualmente as mulheres na fase do climatério foi uma oportunidade ímpar enquanto estudante da graduação, pois dificilmente teria vivenciado tal situação se estivesse em um contexto não pandêmico. Essa experiência me fez refletir sobre o quanto é relevante explorar os benefícios que as ferramentas tecnológicas podem oferecer no âmbito da saúde, no sentido de facilitar a manutenção do cuidado de uma maneira inovadora. Além disso, as avaliações remotas possibilitaram uma maior sensibilidade para identificar como os fatores sociais, ambientais e emocionais da vida das mulheres podem influenciar na coleta de informações, destacando, dessa maneira, a relevância de uma avaliação individualizada pautada na humanização da atenção à saúde" (D1).

"É notório que a oportunidade amplificou minha capacidade de analisar, refletir e buscar informações sobre a população estudada, visto que foi uma experiência acadêmica transformadora. Durante as avaliações, o ambiente foi um facilitador por permitir a realização da atividade e uma barreira, pois nem sempre tínhamos controle da conexão da internet ou do espaço. Também foi perceptível que muitas mulheres desconheciam partes do corpo e suas determinadas funções. $\mathrm{O}$ assoalho pélvico, por exemplo, foi pontuado como uma região desconhecida por muitas, sendo a educação popular dialógica extremamente importante nesses momentos”(D2).

Assim, vemos que as avaliações propiciariam a aquisição de conhecimentos e habilidades acerca da utilização de ferramentas tecnológicas como estratégias facilitadoras na atenção à saúde. Somado a isso, essa experiência aproximou os acadêmicos à comunidade e aos serviços de telessaúde, que vem ganhando destaque devido às restrições impostas pela pandemia da COVID-19.

Ainda, durante as avaliações remotas, os discentes identificaram que a maioria das mulheres climatéricas possuem lacunas em diversos temas importantes para sua saúde. Foi percebido que existe uma compreensão insuficiente da percepção corporal, por exemplo, com relação ao assoalho 
pélvico e sua capacidade de contração. Fortalecendo, dessa forma, a importância da educação em saúde para esta população, a fim de promover o autoconhecimento sobre o corpo feminino. Além disso, a literatura reporta que a implementação de um programa educacional possibilita a redução das disfunções dos músculos do assoalho pélvico, do mesmo jeito, que proporciona uma maior autonomia e melhor qualidade de vida (BERZUK, K., SHAY, B., 2015; FANTE, et. al., 2019).

Em relação à percepção das discentes quanto às respostas das mulheres sobre a fisioterapia, foi notado que uma parcela importante das entrevistadas eram sedentárias e apresentavam comorbidades associadas. Ademais, foi visto que os principais aspectos referidos pelas participantes foram: a diminuição da libido em conjunto com a irritabilidade acentuada e ansiedade. Logo, isso corrobora com os achados pautados em intervenções baseadas na prática de exercício físico ou yoga, os quais desencadeiam efeitos positivos na saúde mental e sexual de mulheres que estão no climatério, assim como, diminui o risco de desenvolvimento de doenças crônicas (SHEPHERD-BANIGAN, M et al., 2017; NGUYEN, et. al, 2020). Ratificando ainda a importância da continuidade e efetiva realização do nosso projeto de pesquisa e extensão.

Portanto, pode-se inferir que as vivências digitais possibilitaram a ampliação do olhar clínico e senso crítico-reflexivo dos acadêmicos de fisioterapia, além do saber lidar com as especificidades de cada participante em seu meio domiciliar, social e cultural distintos, contribuindo efetivamente para uma formação acadêmica e profissional sólida, mesmo diante os desafios impostos pela atual situação pandêmica que estamos vivendo.

\section{SOBRE AS PERSPECTIVAS DAS DISCENTES DE NUTRIÇÃO}

Estiveram presentes também duas discentes do curso de Nutrição, revezando-se entre as avaliações nutricionais virtuais junto com as da Fisioterapia. A vivência dessas, mostra que trouxe a percepção do quanto as estratégias de cuidado à saúde são ampliadas e são uma boa alternativa perante época de pandemia, por reduzir a circulação das mulheres e, por conseguinte, reduzir os riscos de contaminação.

Abaixo, seguem os relatos dos discentes referentes à experiência de avaliação virtual:

"Foi uma experiência que me proporcionou bastante enriquecimento. A cada entrevista nos deparamos com algumas limitações e realidades distintas. A vivência me permitiu ampliar meus conhecimentos a respeito da utilização de ferramentas virtuais voltadas para o cuidado com a saúde, e ainda, ver que pessoas de locais distintos conseguiam participar se sentindo assim mais próximas, 
mesmo que residindo em locais mais distantes da universidade. Ademais, foi possível observar que algumas mulheres se apresentavam mais seguras, já outras se mostravam mais tímidas para responder determinadas perguntas. Também percebi que as participantes se sentiam à vontade para sanar dúvidas a respeito da fase climatérica e, além disso, era notório o desejo delas de cuidarem da saúde e buscarem conhecimentos para passarem pelo climatério de forma mais saudável." (D3);

"Foi a primeira vez que tive a oportunidade de aplicar um questionário sobre consumo alimentar e essa experiência me proporcionou um grande crescimento, tanto no âmbito pessoal como no profissional. Foi muito bom ter tido esse contato, mesmo que de forma virtual, com as mulheres. A cada encontro, tínhamos a chance de conhecer um pouco sobre a vida e a história das climatéricas. Algumas foi preciso remarcar várias vezes, devido a imprevistos ou falhas na conexão por parte das participantes, mas no geral, foi possível notar o grande interesse das mulheres em adquirir conhecimento e melhorarem sua qualidade de vida. Notei que elas se sentiam seguras e abertas para dialogar conosco, algumas gostavam de se estender nas respostas, quando existiam dúvidas, elas sempre perguntavam, ou seja, conseguimos criar uma relação de intimidade e isso contribuiu para o sucesso das entrevistas." (D4).

Os relatos das experiências das avaliações foram colhidos por meio de um grupo de WhatsApp, após as experiências em questão.

Os discursos mostram alguns pontos positivos, como a abrangência de alcance de mulheres que residem em locais distantes e que teriam dificuldades em participar das atividades de forma presencial. Da mesma forma, viram que as participantes se sentiam bem seguras e cômodas, ainda mais por estarem em seus ambientes domiciliares. A cada vídeochamada o contexto era diferente, o que permitia a oportunidade de exercitar a empatia e a resiliência dos discentes. Segundo Cunha et al. (2021, p. 178), o desenvolvimento de habilidades de interação social é tido como um produto ímpar da prática extensionista.

Entretanto, pôde-se perceber algumas limitações, a internet foi um fator que, de certa forma, influenciou as avaliações. Muitas mulheres não tinham acesso a uma rede de qualidade ou não conseguiam manusear a plataforma usada para a avaliação virtual por falta de experiência. O problema em questão pôde ser solucionado com explicações dos próprios avaliadores de como utilizar a plataforma e, nos casos de ausência de internet, as avaliações eram feitas via telefone, para dar seguimento ao projeto e não haver prejuízos.

Em relação à percepção das discentes quanto às respostas das mulheres quanto à nutrição, foi notado que a maioria delas já tinha conhecimento do que era uma alimentação saudável. Estas 
já possuíam alguns hábitos benéficos, e as que não possuíam, tinham consciência e demonstravam vontade de mudar. Segundo Hoffmann et. al., (2015, p. 1570) as mulheres normalmente adquirem hábitos alimentares mais saudáveis com o aumento da idade e isso foi bem visto durante as entrevistas. Grande parte das participantes adotaram uma alimentação mais saudável devido às doenças adquiridas ao longo da vida e por isso já tinham tido um acompanhamento nutricional em algum momento.

Por fim, pode-se inferir que a vivência das avaliações foi importante para o conhecimento das discentes de nutrição sobre promoção de saúde em meio virtual, possibilitando a formação de um senso crítico e empático. Ademais, é perceptível que as mulheres em fase climatérica necessitam de mais atenção à saúde, e a referida experiência levou as participantes a refletir e promover sua autonomia no autocuidado. Desta forma, destacamos o quanto a alimentação saudável e adequada é capaz de influenciar e amenizar os sintomas do climatério, juntamente com a prática regular de exercício físico.

\section{A AVALIAÇÃO VIRTUAL E O CONTEXTO DE EXTENSÃo}

Dentro do contexto de extensão universitária, a avaliação virtual permitiu aos discentes a adesão de uma determinada postura. A experiência foi muito além do conteúdo teórico-prático, isto é, os alunos passaram a reconhecer a linguagem popular, adequando seus conhecimentos científicos a algo mais acessível, sendo esta um instrumento de empoderamento social (MACEDO, SILVA, NASCIMENTO, 2019).

Da mesma forma, o experimento serviu para ampliar os conceitos de educação em saúde dos envolvidos no projeto. A troca de informações estabelecida entre o avaliador e a participante foi muito rica, sabendo que a cultura, os fatores ambientais e sociais influenciavam no saber teórico de cada indivíduo. O resultado da vivência permitiu a construção do modelo dialógico, assim como a integralidade, representando os saberes populares e o senso reflexivo acadêmico (ALMEIDA; MOUTINHO; LEITE, 2016).

\section{CONCLUSÃO}

Conclui-se que a experiência foi válida para os discentes por ser algo novo e proveitoso, ressaltando assim a continuidade das atividades de extensão e pesquisa à medida em que é benéfica tanto para o contexto acadêmico, quanto para o social. Destaca-se também a alternativa como forma de prevenção do contágio da COVID-19, sabendo que todas as participantes e os avaliadores esta- 
vam no conforto de suas residências, sem nenhum contato físico, portanto, sem riscos relacionados à pandemia. Por fim, acredita-se que a modalidade de avaliação virtual será levada para os âmbitos profissionais dos discentes, por ser prática oportuna para as análises dialógicas e adequada para todos os ciclos de vida.

\section{REFERÊNCIA}

ALMEIDA E.R.; MOUTINHO C.B, LEITE M.T.S. Family health nurses' teaching practice in the health education development. Interface (Botucatu). v. 20, $\mathrm{n}^{\circ}$. 57, p. 389-401, 2016. Disponível em <http://dx.doi.org/10.1590/1807-57622015.0128>. Acesso em 05/08/2021.

BERZUK, K., SHAY, B. Effect of increasing awareness of pelvic floor muscle function on pelvic floor dysfunction: a randomized controlled trial. Int Urogynecol J. v. 26, p. 837-844, 2015. Disponível em: https://doi-org.ez18.periodicos.capes.gov.br/10.1007/s00192-014-2599-z. Acesso em: 06/07/2021.

BRASIL. Ministério da Saúde (MS). Secretaria de Atenção à Saúde. Departamento de Ações Programáticas Estratégicas. Direitos Sexuais e Direitos Reprodutivos. Manual de atenção à mulher no climatério/ menopausa. Brasília: MS; 2008. [Série A. Caderno $n^{\circ}$ 9. Normas e Manuais Técnicos]. Acesso em: 08/08/2021.

CONTE, F. A.; FRANZ, L. B. B. Estado nutricional e de saúde de mulheres pós-menopausa. Saúde, Santa Maria, v. 41, n. 1, p.85-92, Jan./Jul 2015. Disponível em: http://dx.doi. org/10.5902/2236583412837. Acesso em: 07/08/2021.

CUNHA, B.S. et al. Projeto Radiologia na Comunidade: relato de experiência de educação em saúde. Revista Extensão em Foco, Palotina, n.22, p. 171-181, jan/jun. 2021. Disponível em: https:// revistas.ufpr.br/extensao/article/view/74263/pdf. Acesso em: 02/08/2021.

DINIZ, E.G.M et al. A extensão universitária frente ao isolamento social imposto pela COVID-19. Braz. J. of Develop. Curitiba, v. 6, n. 9, p. 72999-73010, set. 2020. Disponível em: DOI:10.34117/ bjdv6n9-669. Acesso em: 01/08/2021.

FANTE, J.F. et al. Do Women have Adequate Knowledge about Pelvic Floor Dysfunctions? A Systematic Review. Rev Bras Ginecol Obstet. v. 41, p. 508-519, 2019. Disponível em: DOI https:// doi.org/10.1055/s-0039-1695002. Acesso em: 06/07/2021.

FERREIRA, C.H.J et al. A guide to physiotherapy in urogynecology for patient care during the COVID-19 pandemic. Int Urogynecol J, v. 32, n. 1, p. 203-210, jan. 2021. Disponível em: DOI: 10.1007/s00192-020-04542-8. Acesso em: 06/08/2021.

GUSSO, H.L et al. Ensino superior em tempos de pandemia: diretrizes à gestão universitária. Educ. Soc. Campinas, v. 41, p. 1-27, 2020. Disponível em: https://doi.org/10.1590/ES.238957. 
Acesso em: 01/08/2021.

HOFFMANN, M. et al. "Padrões alimentares de mulheres no climatério em atendimento ambulatorial no Sul do Brasil”. Ciência \& Saúde Coletiva, Rio de Janeiro, v. 20, n. 5, p. 1565-1574, Mai. 2015. Disponível em: https://doi.org/10.1590/1413-81232015205.07942014. Acesso em: $02 / 08 / 2021$.

KAYGISIZ, B.B; TOPCU, Z.G; MERIÇ, A; GÖZGEN, H; ÇOBAN, F. Determination of exercise habits, physical activity level and anxiety level of postmenopausal women during COVID-19 pandemic. Health Care for Women International, v. 41, p.1240-1254, dez. 2020. Disponível em: DOI: 10.1080/07399332.2020.1842878. Acesso em: 07/08/2021.

MACEDO, A.M.R.M; SILVA, E.R; NASCIMENTO, J.S. "Linguagem Culta x Linguagem Popular nas Aulas de Língua Portuguesa da Educação de Jovens e Adultos EJA: Desafios e Possibilidades". Revista Porto das Letras, v. 5, nº. 1, p. 85-101, Janeiro de 2019. Disponível em: https://sistemas.uft.edu.br/periodicos/index.php/portodasletras/article/view/4883/14837. Acesso em: 02/08/2021.

MAGAlHÃES, A. G.; SILVA, C. A. M.; PEREIRA, I. N.; DA SILVA, V. F. D.; EUFRÁSIO, L. S. Percepção discente frente à estratégia de telemonitoramento em tempos de pandemia, 2020. Revista Extensão \& Sociedade, v. 12, n. 1, 4 set. 2020. DOI: https://doi.org/10.21680/2178-6054.2020v12n1ID20747.

MARTINES, R. S.; MEDEIROS, L.M.; SILVA, J.P.M.; CAMILLO, C.M. O uso das TICs como recurso pedagógico em sala de aula. In: Congresso Internacional de Educação e Tecnologias. Encontro de Pesquisadores em Educação à Distância. Educação e Tecnologias: inovação em cenários em transição. 2018. p.1-12.

MATA, K.R.U et al. Telehealth in the rehabilitation of female pelvic floor dysfunction: a systematic literature review. Int Urogynecol J, v. 32, p. 249-259, nov. 2020. Disponível em: DOI: 10.1007/s00192-020-04588-8. Acesso em: 06/07/2021.

MONTILLA, R. N. G.; ALDRIGHI, J. M.; MARUCCI, M. F. N. Relação cálcio/proteína da dieta de mulheres no climatério. Revista da Associação Médica Brasileira, São Paulo, v. 50, n. 1, p. 52-44, 2004. Disponível em: https://doi.org/10.1590/S0104-42302004000100035. Acesso em: 06/07/2021.

NGUYEN, T.M; DO, T.T.T; TRAN, T.N; KIM, J.H. Exercise and Quality of Life in Women with Menopausal Symptoms: A Systematic Review and Meta-Analysis of Randomized Controlled Trials. Int J Environ Res Public Health. v. 17, no. 19, p. 7049, set/ 2020. Disponível em: DOI:10.3390/ijerph17197049. Acesso em: 06/08/2021.

NIENHUIS C.P, LESSER I.A. The Impact of COVID-19 on Women's Physical Activity Behavior and Mental Well-Being. Int J Environ Res Public Health, v. 17, n. 23, p.1-12, dez. 2020. Disponível em: doi:10.3390/ijerph17239036. Acesso em: 06/08/2021. 
SHEPHERD-BANIGAN, M et al. Improving vasomotor symptoms; psychological symptoms; and health-related quality of life in peri- or post-menopausal women through yoga: An umbrella systematic review and meta-analysis. Complementary therapies in medicine. v. 34, p. 156-164, 2017. Disponível em: DOI:10.1016/j.ctim.2017.08.011. Acesso em: 06/08/2021. 